\title{
Effect of lidocaine on bone matrix formation by osteoblasts
}

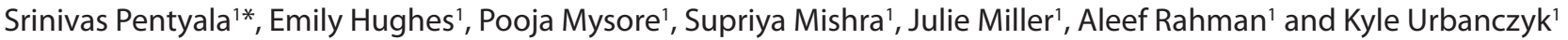

\begin{abstract}
Local anesthetics are widely used as an intraosseous injection particularly in the field of dentistry. Occasionally, large doses of anesthetics are injected to elicit the desired effect during many surgical procedures. In this study we examined the effect of a local anesthetic, lidocaine, on osteoblasts as there are only a few reports on the effect of local anesthetics directly on bone cells. Our results showed that physiological as well as supra physiological concentrations of lidocaine cause cell death. At low concentrations, lidocaine was found to enhance both proliferation of osteoblasts and bone matrix production. In addition, low concentration of lidocaine increased the expression of osteocalcin, a key regulator in the signaling pathway of bone matrix production. Our results show that lidocaine has a biphasic effect on osteoblasts and low concentrations of this anesthetic may assist in bone matrix production. This preliminary study can lead to the therapeutic usage of intraosseous lidocaine as a bone strengthening agent.
\end{abstract}

\section{Introduction}

Local anesthetics have been delivered via the intraosseous (IO) route for decades. Injecting an anesthetic by $I O$ route gains vascular access into the marrow cavity where an intravenous line is placed in order to administer medications and fluid into the bone marrow. Lidocaine is one of the most commonly used local anesthetics that are used in IO procedures and occasionally, large doses of anesthetic are delivered in order to produce the desired effect [1,2]. This method was previously employed in the early twentieth century until the introduction of plastic catheters. This made the placement of intravenous needles much easier thereby causing physicians to favor the IV route. Even so, placing a central line remains extremely invasive and often requires ultrasound guidance to do accurately in osteoporotic patients [3], that are dehydrated or in poor health. 10 access, on the other hand, is relatively easy to obtain, even under adverse clinical conditions. For this reason, IO made a comeback in the past thirty years particularly in pediatric emergencies. 10 injection is now gaining popularity for use in regional anesthesia in treating osteoporotic fractures and even management of hydrocephalus $[4,5]$ and is making resurgence as an option for vascular access during resuscitation when peripheral venous access is not available.

Osteoporosis is a disease that affects over ten million people in the United States alone, and over 75 million in the United States, Europe, and Japan combined. It is devastating to patients and pricey to an already overburdened healthcare system, costing over \$14 billion per year in only the United States. Its incidence is rising with age expectancy and consequently becoming a major public health issue that needs to be addressed more effectively [6].

*correspondence: srinivas.pentyala@stonybrook.edu Department of Anesthesiology, Stony Brook Medical Center, Stony Brook, NY 11794-8480.

Full list of author's information is available at the end of the article
The hallmarks of osteoporosis are reduced bone mineral density (BMD) and disrupted bone micro architecture. The pathophysiological cause of osteoporosis is essentially an imbalance in bone metabolism. In normal individuals, bone constantly undergoes remodeling. Osteoclasts resorb bone, and osteoblasts fix circulating calcium into new bone. It is estimated that nearly $10 \%$ of bone mass is undergoing remodeling at all times [7]. In individuals with osteoporosis, however, the problem is thought to be three-fold-peak bone mass is inadequate compared to that of normal individuals, osteoclasts are excessively active, and osteoblast activity is insufficient to keep up with bone resorption.

Most drug treatments for osteoporosis pursue only a one-dimensional approach in that they are antiresorptive but fail address the need to build bone. Bisphosphonates were discovered in the nineteenth century but were not used until the 1960s for treatments of osteoporosis, and remain the first line treatment. Bisphosphonates work by encouraging osteoclast apoptosis, and are often taken with calcium. While relatively effective, these drugs produce gastrointestinal inflammation that is often intolerable to patients. In rare cases, these agents also cause osteonecrosis of the jaw [8,9].

Hormones are sometimes prescribed for treating osteoporosis. Estrogen may be prescribed in postmenopausal women and testosterone in hypogonadal men $[10,11]$. This treatment is controversial, and while mechanistically hormone replacement should help prevent the disease, no conclusive clinical studies have shown its effectiveness. Selective Estrogen Receptor Modulators (SERMS), particularly Raloxifene, appear to slow bone resorption and have proven effective for prevention of osteoporosis [12]. Finally, calcitonin directly inhibits osteoclast activity via the calcitonin receptor. Its mode of action is to disrupt formation of the actin cytoskeleton in osteoclasts [13]. In addition, only one true anabolic agent is on the market, and is typically relegated for use only if bisphosphonates have failed [14]. Teriparatide (Forteo), which is a recombinant parathyroid hormone (PTH) residue 1-34, acts like 
Pentyala et al. Journal of Anesthesiology and Clinical Science 2012,

http://www.hoajonline.com/journals/pdf/2049-9752-1-1.pdf

doi: $10.7243 / 2049-9752-1-1$

PTH and stimulates osteoblasts. Unfortunately, clinical trials have suggested that it increases risk of osteosarcoma.

Since the IO route delivers medication and fluids systemically as rapidly as the IV route, it appears to be an ideal alternative when IV access is difficult to obtain. There are only a few studies performed on the effects of IV anesthetics directly on bone cell, most of which have only described cytotoxic effects of drugs on osteoblasts $[15,16]$. There was only one report in literature showing differential effect of richlocaine on osteoblasts and bone matrix production [17] which may have a direct impact in developing novel therapies for the treatment of bone related disorders like Osteoporosis. For this reason, it is crucial to investigate the possible effects of drugs delivered via the IO route. In this study we report the differential effects of lidocaine on osteoblast cell proliferation and bone matrix formation.

\section{Materials \& Methods 1. Cell Culture}

A human fetal-osteoblast cell line, hFOB 1.19, established by Harris et al [18]. which displays osteoblast-specific phenotypic markers and mineralizes extracellular matrix (ECM) was used in these studies. This cell line has minimal chromosome abnormalities and expresses a normal spectrum of matrix proteins [19]. hFOB cells were procured from ATTC (American type culture collection, Rockville, MD, USA) and were cultured at $34^{\circ} \mathrm{C}$ in a 1:1 mixture of Dulbecco's Modified Eagle Medium DMEM (high glucose) and Hams F12 supplemented with $15 \mathrm{~mm}$ Hepes, $0.5 \mathrm{~mm}$ Na Pyruvate, $1.2 \mathrm{~g} / \mathrm{l}$ Na bicarbonate, $0.3 \mathrm{mg} /$ $\mathrm{ml} \mathrm{G} 418,10 \%$ fetal calf serum (FCS), $100 \mathrm{U} / \mathrm{ml}$ penicillin and $10 \mathrm{mg} /$ $\mathrm{ml}$ streptomycin in a humified atmosphere of $5 \% \mathrm{CO}_{2}$.

\section{Lidocaine Studies}

I. Effects of lidocaine on osteoblast proliferation and bone matrix formation Osteoblasts were grown at $34^{\circ} \mathrm{C}$ to $30-40 \%$ confluency and treated with $1 \%$ lidocaine hydrochloride in different concentrations $(0-10 \mathrm{mM})$. The treated cells were propagated for 72 hours at $34^{\circ} \mathrm{C}$ until they reached nearly $90 \%$ confluence in the control set. The plates were then rinsed with PBS and fixed with $70 \%$ Ethanol. The presence of mineralized nodules was confirmed using alizarin red S staining. $2 \%$ alizarin red S (Sigma) was prepared in distilled water and the $\mathrm{pH}$ was adjusted to $4.1-4.3$ using $0.5 \%$ ammonium hydroxide. Ethanol fixed cultures were washed with PBS, and stained with alizarin red $S$ for 10-15 min. After removal of unincorporated excess dye with distilled water, the cells were examined using phase-contrast and fluorescence microscopy with an Olympus IMT-2 inverted microscope (Olympus, NJ) equipped with a $100 \mathrm{~W}$ high-pressure mercury lamp.

\section{Effects of lidocaine on osteocalcin}

Osteoblasts grown to $30 \%$ confluency were treated with different concentrations of lidocaine. After incubating cells at $34^{\circ} \mathrm{C}$ for 48 hours, they were washed once with PBS and lysed using sigma lytic buffer. Cell lysates were subjected to SDS-PAGE and the proteins are transferred to PVDF membrane. The membrane is blocked in 5\% Dry Milk in TBS and probed with a polyclonal osteocalcin antibody. The blot is then probed with goat anti-rabbit lgG, labeled with HRP. The blot is developed in ECL reagent (Amersham) and scanned. Luminescence intensities are quantified and presented in a graphical form.

\section{Results}

\section{Effects of lidocaine on osteoblasts}

The first cell treatments with lidocaine ranged in doses from $2 \mathrm{mM}$ to $10 \mathrm{mM}$. It was anticipated that microscopic examination would show cytotoxicity and cell death in proportion with the concentration of lidocaine. Instead, the cells treated with $2 \mathrm{mM}$ lidocaine showed increased proliferation as well as increased bone matrix production compared to the control. Cells treated with $5 \mathrm{mM}$ lidocaine showed the same result compared to the control, but less proliferation and bone matrix production than cells treated with $2 \mathrm{mM}$ lidocaine (Figure 1). Cells did not survive $10 \mathrm{mM}$ lidocaine treatment (Figure 2). Due to these unexpected results, this experiment was repeated multiple times with more incremental concentrations in an attempt to establish an approximate EC50 at which cells benefited the most from lidocaine treatment as reflected by increased bone matrix production. The number of cells per field was counted and a 3 fold increase of cell proliferation was observed with $2 \mathrm{mM}$ lidocaine when compared to control and a 2 fold increase was observed with $5 \mathrm{mM}$ lidocaine (Figure 3$)$. Higher concentration of lidocaine $(10 \mathrm{mM})$ was found to be toxic to the cells. Images taken at high concentrations of lidocaine showed vacuole formation and membrane blebbing preceding apoptosis and later nuclear fragmentation (Figure 4).

\section{Effects of lidocaine on osteocalcin}

Cell extracts treated with and without lidocaine were subjected to SDS-PAGE and immunoblot for osteocalcin expression was performed. Osteocalcin expression was found to increase in cells treated with $2 \mathrm{mM}$ lidocaine and quantification of the pixel intensities of the osteocalcin signal showing a 1.6 times increase in the levels of osteocalcin expression confirmed this observation (Figure 5).

\section{Discussion}

IO injection is making resurgence and is now gaining popularity for use in regional anesthesia [4,5]. Yet, there are only a few reports on the direct effect of local anesthetics on bone. Our results show that lidocaine at low concentrations enhanced the proliferation of osteoblasts and also increased the bone matrix production by these cells. These observations can lead to the therapeutic usage of intraosseous lidocaine as a possible bone strengthening agent. Since bone is continuously degraded and regenerated by a cycle of osteoclasts and osteoblasts, the disease of osteoporosis calls for an immediate solution to bone regeneration, not just 


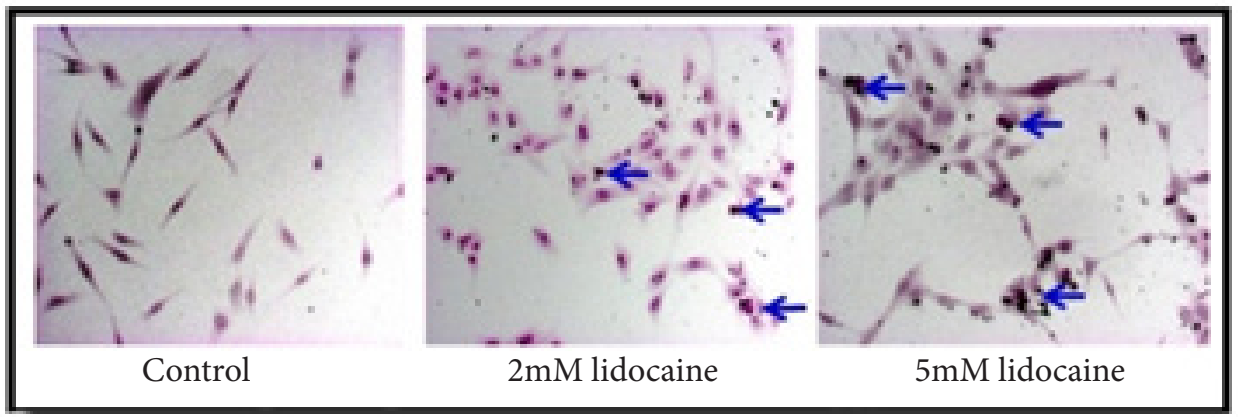

Figure 1: Human fetal osteoblast cells (hFOB 1.19) were grown at $34^{\circ} \mathrm{C}$ using Dulbecco's modified Eagle medium-Ham's F-12. Once the cells became $30-40 \%$ confluent, they were treated with $1 \%$ lidocaine hydrochloride. The treated cells were propagated for 72 hours at $34^{\circ} \mathrm{C}$ until they reached nearly $90 \%$ confluence in the control set. The plates were then rinsed with PBS and fixed with $70 \%$ Ethanol. Staining with Alizarin Red dye was done, which preferentially binds bone matrix, and then cells were examined using phase-contrast and fluorescence microscopy. Microscopy images of hFOB 1.19 cells left untreated (left), treated with 2 mM lidocaine (middle) and 5 mM lidocaine (right). Cells proliferated and exhibited increased bone matrix production with low treatment of lidocaine ( $2 \mathrm{mM})$. With $5 \mathrm{mM}$ lidocaine, there was increased proliferation compared to the control, but considerably less than at $2 \mathrm{mM}$ lidocaine.

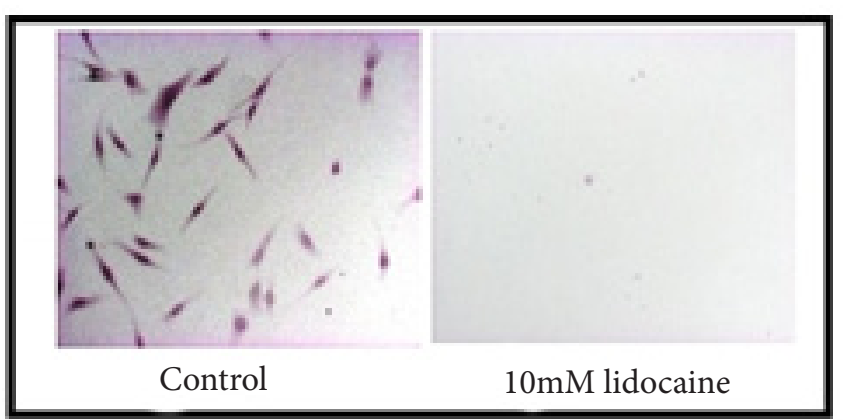

Figure 2: hFOB 1.19 cells were grown at $34^{\circ} \mathrm{C}$ using Dulbecco's modified Eagle medium-Ham's F-12 and treated with 1\% lidocaine hydrochloride. Microscopy images of hFOB 1.19 cells left untreated (left), treated with $10 \mathrm{mM}$ lidocaine (right).

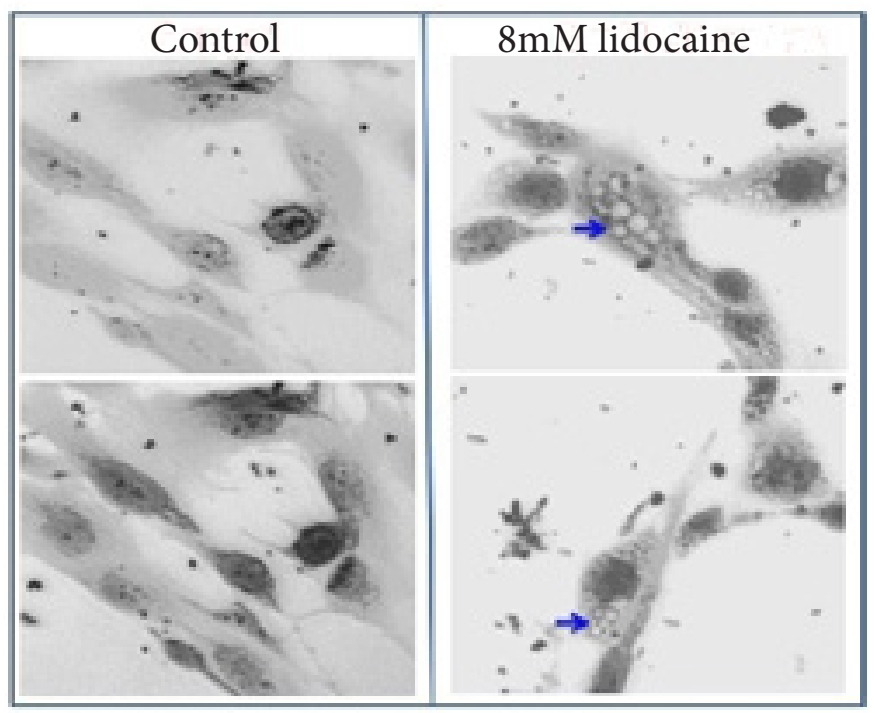

Figure 4: hFob 1.19 cells were treated with $1 \%$ lidocaine hydrochloride and were propagated for 72 hours at $34^{\circ} \mathrm{C}$ until they reached nearly $90 \%$ confluence in the control set. Membrane blebbing and vacuole formation were visible in the cells treated with $8 \mathrm{mM}$ lidocaine, preceding apoptosis.

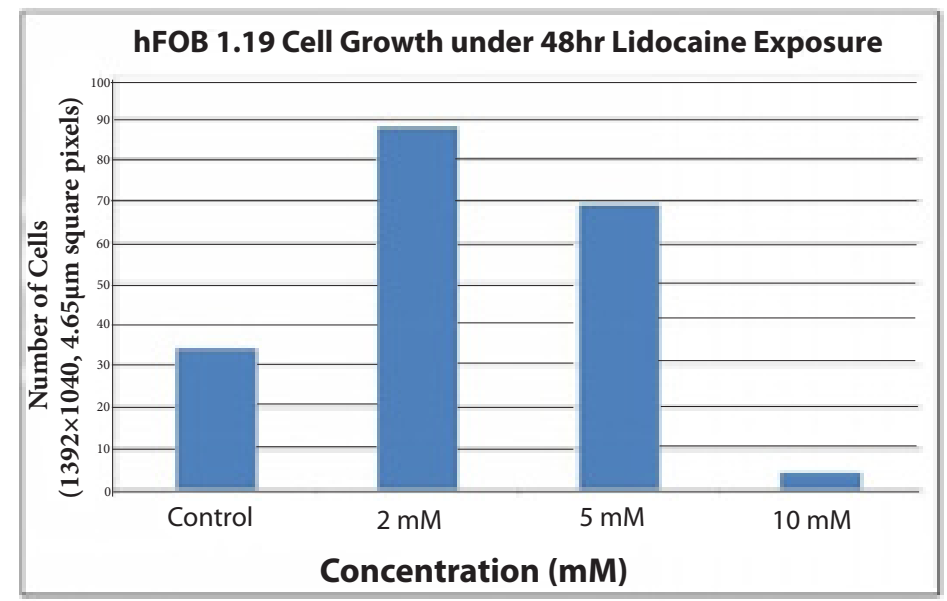

Figure 3: hFOB 1.19 cells were treated with incremental concentrations of lidocaine from $2 \mathrm{mM}$ to $10 \mathrm{mM}$ Lidocaine (right). Cells treated with minimal Lidocaine $(2 \mathrm{mM}$ ) exhibited the most bone matrix proliferation when $90 \%$ confluency was reached. 


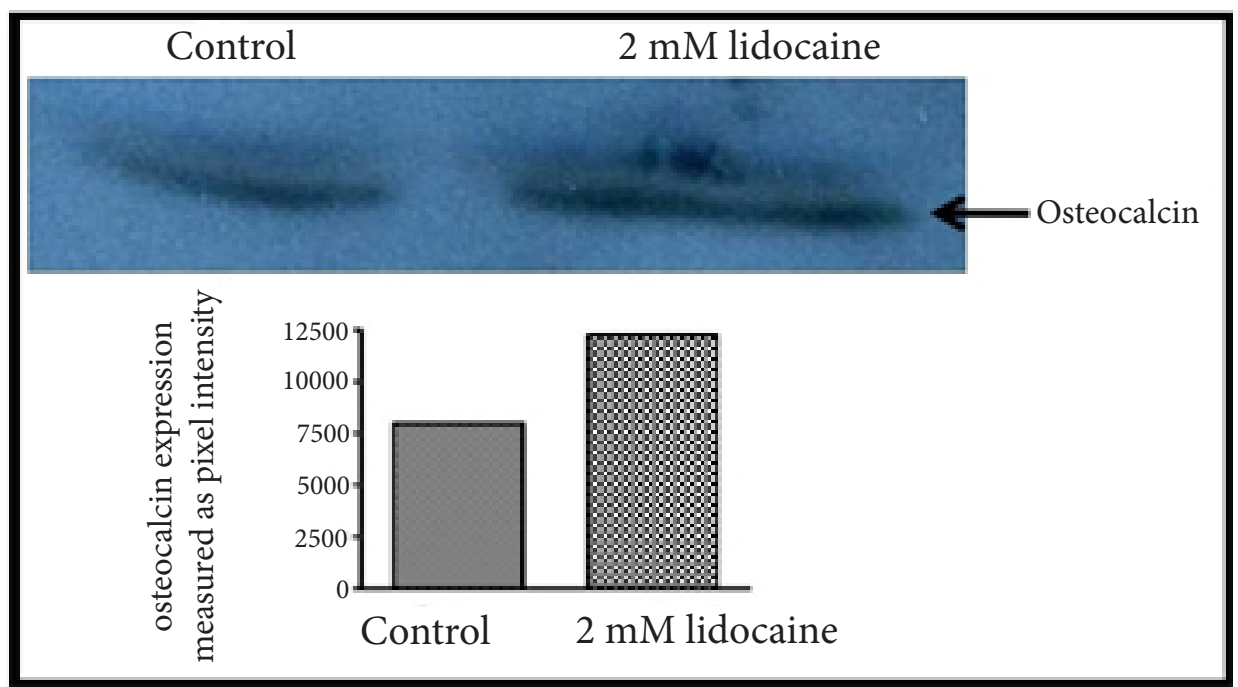

Figure 5: hFOB 1.19 cells were plated, grown to $30 \%$ confluency, and treated with lidocaine. Control plates were left untreated, and the remaining plates were treated with $2 \mathrm{mM}$ lidocaine. After incubating cells at $34^{\circ} \mathrm{C}$ for 48 hours, they were washed once with PBS and lysed using sigma lytic buffer. Samples from the control and treated sets of cell lysates were subjected to SDS-PAGE. Resolved proteins were transferred to PVDF and immunoblotting was performed with osteocalcin antibody and visualized by ECL (G.E.). In the cells treated with low concentrations of lidocaine, an increased osteocalcin expression was observed. The increase in osteocalcin expression in cells treated with $2 \mathrm{mM}$ lidocaine was confirmed by quantification of the pixel intensities of the scanned X-ray film.

the halting of bone degradation. Current research reveals that biocompatible fibroins for bone regeneration is still a potential tool for bone regeneration, but is a long way off from being implemented as a possible treatment [20], while other research has examined substances such as taurine, which increases $h F O B$ cell proliferation [21]. In addition a therapeutic concentration of 2,6-diisopropylphenol has been shown to protect osteoblasts from sodium nitroprusside and hydrogen peroxide-induced cell insults [22]. Discovery of a new anabolic drug for bone will have tremendous impact in the treatment of bone related disorders.

Local anesthetics have been given via IO for decades, and lidocaine is one of the most commonly used local anesthetics. Numerous studies have been published about the cytotoxic effects of local anesthetics on cells, including osteoblastic cells $[15,16]$. We initiated this study to evaluate the toxic effects of lidocaine on osteoblasts. Interestingly it was observed that cells appeared to not only thrive, but to also proliferate and produce more bone matrix at low doses of lidocaine (Figure 1).

Extensive literature review revealed only one paper that supported this observation. In vitro studies on osteoblastic cells and in vivo studies in rats were performed with richlocaine, and proliferation was reported [17]. Richlocaine is an approved local anesthetic in Russia but not used in Europe and America. Though it is unclear why further studies were not done, this finding may hold great promises for clinical use. Both richlocaine and lidocaine belong to the "amide" class of local anesthetics and most of the studies on osteoblasts with local anesthetics were performed with "ester" class of drugs (Cocaine, procaine, and tetracaine). All these ester class drugs were shown to be toxic to osteoblasts even at low range of concentrations $[15,17,23]$. Amide drugs might interact differentially with osteoblasts at low concentration by activating signaling pathways (as shown by induction of osteocalcin) while ester drugs might trigger the apoptotic pathways. Future studies trying to differentiate between ester and amide local anesthetics effects might throw light on structure specific activity of these drugs on osteoblasts. If local anesthetics have a proliferative effect on both osteoblast growth and matrix secretion, they may potentially be used as a new form of treatment for bone health maintenance. These treatments can target bone pain, bone remodeling in patients suffering from osteoporosis, fractures, and possibly other osteoid diseases.

Though this is a preliminary stage of investigation, it looks promising that local anesthetics may hold the key to someday being used in conjunction with other treatment regimens to promote bone regeneration. Most treatments for osteoporosis are antiresorptive but do not address the need to build new bone. It would be highly beneficial to see whether low doses of lidocaine in conjunction with other bone anabolic agents like Teriparatide (marketed as Forteo by Eli Lilly) can show a synergistic effect in bone rebuilding. In view of the increased interest in 10 route of administering local anesthetics, new findings of the cellular and molecular effects of these drugs on bone cells would help in developing novel modalities of treatments for bone related disorders. 


\section{Article History}

Editor: J.E. Tetzlaff, Case Western Reserve University, USA. Received: 15-Dec-2011 Revised: 12-Jan-2012

Re-Revised: 20-Jan-2012 Accepted: 15-Feb-2012

Published: 12-Mar-2012

\section{Author Information}

'Department of Anesthesiology, Stony Brook

Medical Center, Stony Brook, NY.

\section{Acknowledgement \& Funding}

All the authors are affiliated with Department of Anesthesiology at Stony Brook Medical Center, we did not separately acknowledge this.

\section{Conflict of interest}

There is no conflict of interests for all the authors who worked on this project.

\section{References}

1. Prengel, A. W. et al. [What's new in cardiopulmonary resuscitation? American Heart Association]. Anaesthesist 43, 309-315

2. Zaritsky, A. Pediatric resuscitation pharmacology. Members of the Medications in Pediatric Resuscitation Panel. Ann Emerg Med 22, 445-455

3. Dauri $M$, et al. Continuous i.v. infusion of remifentanil and intraosseous lidocaine provide better analgesia than intraosseous lidocaine alone in percutaneous vertebroplasty of osteoporotic fractures. Br J Anaesth 103, 901-902

4. Pugh, J. A. et al. Intraosseous infusion into the skull: potential application for the management of hydrocephalus. J Neurosurg 106, 120-125

5. Sesay, M. et al. Intraosseous lidocaine provides effective analgesia for percutaneous vertebroplasty of osteoporotic fractures. Can J Anaesth 49, 137-143

6. Riggs, B. L. et al. The worldwide problem of osteoporosis: insights afforded by epidemiology. Bone 17, 505S-511S

7. Arnaud: Mineral and bone homeostasis. In: Textbook of Medicine. Philadelphia: WB Saunders Co; 1985: 1415-1423

8. Fojtik, Z. et al. [Bisphosphonates in the treatment of osteoporosis]. Vnitr Lek 43, 696-699

9. Gatti, D. et al. New bisphosphonates in the treatment of bone diseases. Drugs Aging 15, 285-296

10. Shoupe, D. Androgens and bone: clinical implications for menopausal women. Am J Obstet Gynecol 180, S329-333

11. Hodgson, S. F. et al. American Association of Clinical Endocrinologists medical guidelines for clinical practice for the prevention and treatment of postmenopausal osteoporosis: 2001 edition, with selected updates for 2003. Endocr Pract 9, 544-564

12. Mitlak, B. H. et al. In search of optimal long-term female hormone replacement: the potential of selective estrogen receptor modulators. Horm Res 48, 155-163

13. Inzerillo, A. M. et al. Calcitonin: physiological actions and clinical applications. J Pediatr Endocrinol Metab 17, 931-940

14. Orwoll, E. S. et al. The effect of teriparatide [human parathyroid hormone (1-34)] therapy on bone density in men with osteoporosis. J Bone Miner Res 18, 9-17
15. Nakamura, K. et al. Prilocaine induces apoptosis in osteoblastic cells. Can J Anaesth 46, 476-482

16. Perez-Castro, R. et al. Cytotoxicity of local anesthetics in human neuronal cells. Anesth Analg 108, 997-1007

17. Zadorozhnyi, A. V. et al. Effect of richlocaine on proliferative activity of osteoblasts and intracellular calcium content in rats. Bull Exp Biol Med 136, 375-376

18. Harris, S. A. et al. Development and characterization of a conditionally immortalized human fetal osteoblastic cell line. J Bone Miner Res 10, 178-186

19. Subramaniam, M. et al. Further characterization of human fetal osteoblastic hFOB 1.19 and hFOB/ER alpha cells: bone formation in vivo and karyotype analysis using multicolor fluorescent in situ hybridization. J Cell Biochem 87, 9-15

20. Park, S. Y. et al. Electrospun silk fibroin scaffolds with macropores for bone regeneration: an in vitro and in vivo study. Tissue Eng Part A 16, 1271-1279

21. Jeon, S. H. et al. Taurine increases cell proliferation and generates an increase in [Mg2+]i accompanied by ERK 1/2 activation in human osteoblast cells. FEBS Lett 581, 5929-5934

22. Chen, R. M. et al. 2,6-Diisopropylphenol protects osteoblasts from oxidative stress-induced apoptosis through suppression of caspase-3 activation. Ann N Y Acad Sci 1042, 448-459

23. Wang, D. et al. Bupivacaine decreases cell viability and matrix protein synthesis in an intervertebral disc organ model system. Spine J 11, 139-146

\section{Citation:}

Pentyala S, Hughes E, Mysore P, Mishra S, Miller J, Rahman A and Urbanczyk K: Effect of lidocaine on bone matrix formation by osteoblasts. journal of Anesthesiology and Clinical Science 2012, 1:1. http://dx.doi.org/10.7243/2049-9752-1-1 